\title{
EXTRACTION OF THE OPTIC DISK BOUNDARY IN DIGITAL FUNDUS IMAGES
}

\author{
F. Mendels ${ }^{*}$, C. Heneghan ${ }^{* *}$, P. D. Harper ${ }^{* *}$, R. B. Reilly ${ }^{* *}$, and J.-Ph. Thiran ${ }^{*}$ \\ "Signal Processing Laboratory (LTS), Swiss Federal Institute of Technology (EPFL), \\ CH-1015 Lausanne, SWITZERLAND \\ ** Dept. of Electronic and Electrical Engineering, University College Dublin, \\ Belfield, Dublin 4, IRELAND

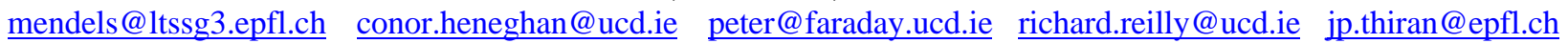

\begin{abstract}
The methods of active contours ("snakes") and level sets were applied to images of the retina in order to locate the outer boundary of the optic disk. A gradient-vector-flow based active contour was used as it performed well over a large range of initial conditions. Images were pre-processed to lessen the influence of blood vessels on boundary detection. Both active contours and level set methods accurately located the correct boundary; level set methods were computationally more intensive.
\end{abstract}

\section{INTRODUCTION}

The optic disk is a significant anatomical landmark in the retina. Various ophthalmic pathologies, especially glaucoma, are manifest by changes in the shape, pallor, or depth of the optic disk region. Accurate identification of the outer boundary of the optic disk may allow ophthalmologists to quantitatively assess changes in the optic disk over time. This paper considers two techniques for locating the outer boundary of the optic disk using color digital images of the retina, a problem which has not previously been addressed.

\section{THEORY}

Active contours ("snakes") have been widely used in the detection of closed contours. These are energy minimizing contours guided by external (image-derived) and internal (contour-derived) forces. Initial formulations of active contours suffered from a need for good initialization, and an inability to move into small concavities. A gradient vector flow (GVF) based snake was introduced to address these limitations [2]. In this formulation, a more general external force is defined which gives a directional field that accounts for boundary proximity, but with a larger range of attraction. This decreases the sensitivity to initial conditions. An alternative approach to boundary detection is offered by level-set theory [3]. In this, a desired propagating boundary is considered as the zero level set of a higher dimensional function $\Psi(x, t)$. The goal is to produce an equation for the evolving function $\Psi(x, t)$ which contains the embedded motion of the boundary as the evolving zero level-set of $\Psi(\boldsymbol{x}, t)$. An evolution equation for $\Psi(\boldsymbol{x}, t)$ can be written, with an explicit "speed" function which controls the time rate of evolution. If the speed on the propagating interface drops close to zero, a convergent boundary is found. A speed function inversely proportional to the image gradient encourages the level set to propagate to strong-edged boundaries in the image. The narrow-band extension is used to increase computational speed [3].
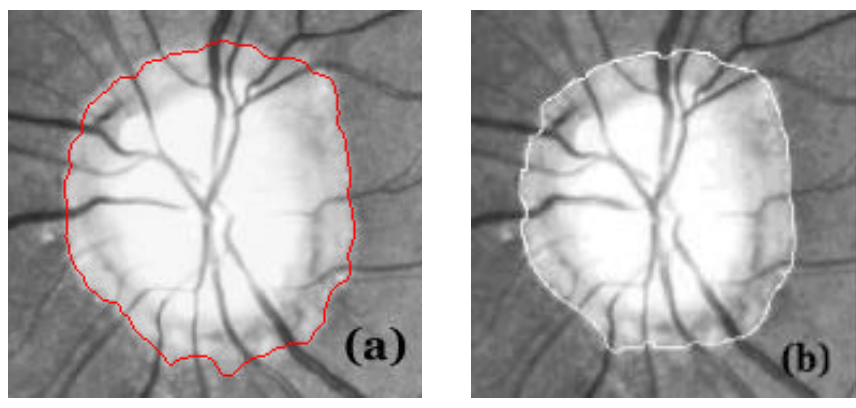

Figure 1: (a) Optic disk boundary located by active contour method. (b) Same boundary using level sets.

\section{RESULTS}

Both methods were applied to sample images $(n=9$, image size $=285 \times 400$ pixels). Conversion from RGB to luminance was carried out prior to boundary detection. Initial experiments showed that the strong edges due to blood vessels crossing the optic disk prevented correct boundary identification. Pre-processing approaches were developed to minimize the confounding effect of these vessels, with the aim of removing the vessel structure from the optic disk. In one technique, candidate vessel pixels were identified as local luminance minima, and replaced with "background" optic disk pixels, if certain conditions were satisfied. In the second approach, morphological operations (dilation, erosion, and maximization) were carried out using a $5 \times 5$-structuring element. Both techniques produced an image on which vessel structures had been largely removed. The optic disk boundary was then located on the processed image using both the GVFbased active contour and level-set algorithms. Sample detected boundaries are shown in Figure 1.

\section{DISCUSSION AND CONCLUSION}

Both techniques detected boundaries which were considered accurate by two clinical ophthalmologists. A disadvantage of the level set method over active contours was its high computational cost. Methods are currently being investigated for improving performance, including the generation of an hierarchical adaptive mesh structure.

\section{REFERENCES}

[1] M. Kass, A. Witkin, D. Terzopoulos "Snakes: Active Contour Models", Int. J. of Computer Vision, vol. 1, pp 321-331, 1988.

[2] C. Xu, J. L. Prince, "Snakes, shapes, and gradient vector flow," IEEE Trans. on Image Processing, vol. 7, pp.359-369, 1998.

[3] J.A. Sethian, "Level Set Methods", Cambridge Monographs on Applied and Computational Mathematics, Cambridge, 1996. 